\title{
Lettre à l'éditeur \\ Conséquences écologiques des accidents nucléaires de Kyshtym et de Tchernobyl (URSS)
}

C. MYTTENAERE*

Les scientifiques européens se sont penchés depuis de nombreuses années sur l'étude des effets de la pollution radioactive sur l'homme et son environnement et ceci grâce, en grande partie, aux subsides alloués par la Commission des communautés européennes (CCE). Cet engouement tire son origine des explosions nucléaires atmosphériques, de l'emploi de l'arme atomique (Hiroshima et Nagasaki, 1945) ainsi que des accidents nucléaires majeurs (Kyshtym, URSS, 1957 ; Windscale, UK, 1957 ; Three-Mile Island, USA, 1979 et Tchernobyl, URSS, 1986). Les conséquences écologiques des accidents nucléaires ont peu retenu l'attention du public jusqu'à ce jour, les effets sur l'homme et les populations faisant surtout la une des médias. Parmi les accidents nucléaires qui ont eu un impact important sur l'environnement, deux retiennent particulièrement l'attention : Kyshtym et Tchernobyl.

Les scientifiques occidentaux n'ont eu connaissance de l'accident de Kyshtym (Oural méridional) qu'en 1976, mais il a fallu attendre la "Glasnost" pour en connaître un peu plus au sujet de l'influence des radionucléides rejetés sur l'environnement proche. Par contre, dès après l'accident de Tchernobyl, les portes de l'information soviétique se sont entr'ouvertes et les renseignements donnés par les autorités soviétiques, les contacts plus étroits et plus fréquents avec nos collègues soviétiques ainsi que les visites effectuées en URSS ont permis de se faire une idée plus précise de l'ampleur des dégâts et des conséquences à long terme des rejets de radioactivité dans le milieu. Au cours des deux dernières années, des chercheurs de la CEE ont eu la possibilité de visiter plusieurs fois les sites contaminés en URSS, dans le cadre des activités de I'Union internationale de radioécologie (UIR). De plus, une conférence internationale organisée par I'UIR et les DG-XI et DG-XII de la CEE à Luxembourg a permis de récolter un ensemble inédit d'informations et de préciser ainsi les conséquences de ces accidents.

\footnotetext{
- Laboratoire de biologie végétale, Université catholique de Louvain, 5, place Croix du Sud, B-1348 Louvain-la-Neuve, Belgique.
} 
Le complexe nucléaire de Kyshtym, aussi appelé Tchelyabinsk-40, est un centre militaire destiné à la production de plutonium. La construction de ce centre a débuté en 1946 et il fut opérationnel en 1948. Dès 1948 des déchets nucléaires ont été déversés dans une rivière proche du centre, la Techa. Suite à la contamination élevée de cet écosystème aquatique, les déchets ont été ensuite déversés (1953) dans un lac de faible superficie, le lac Karachay. Pour les mêmes raisons que précédemment, ils ont enfin été stockés dans des cuves de $300 \mathrm{~m}^{3}$. Ces cuves de stockage nécessitaient, toutefois, un refroidissement continu. En septembre 1957, suite à un problème de refroidissement, une cuve en voie d'évaporation, a explosé : $20 \mathrm{MCi}(740 \mathrm{PBq})$ auraient ainsi été rejetés dans l'atmosphère ; $90 \%$ de cette radioactivité se seraient déposés à proximité du lieu de l'accident et $10 \%$ ont été dispersés sur une surface de $\pm 20000 \mathrm{~km}^{2}$ (provinces de Tchelyabinsk, Sverdlovsk et Tyamen). La zone contaminée en forme de fuseau s'étend sur plus de $300 \mathrm{~km}$ de longueur en direction nord-est. La largeur de la trace varie de 30 à $50 \mathrm{~km}$; 11000 personnes ont été évacuées, 1054 dans les 7 à 10 jours après l'accident et le reste entre 250 et 670 jours. La surface contaminée englobait 217 villages pour une population de 270000 personnes.

II est difficile de se prononcer, à l'heure actuelle, sur les effets de la radioactivité sur la population. Tout au plus, peut-on mentionner que les données en notre possession ne révèlent aucun effet significatif par rapport aux populations témoins. On ne possède, toutefois, aucune donnée concernant les militaires qui ont participé aux travaux de décontamination et à la garde des sites les plus contaminés. L'accident de Kyshtym s'est produit en automne au moment où la faune et la flore entraient en "dormance". La zone contaminée était très fertile et $60 \%$ de celle-ci étaient destinés à l'agriculture, $40 \%$ étant occupés par la forêt et les lacs. Cet accident a donné l'occasion à nos collègues soviétiques d'étudier en grandeur nature l'effet de la contamination radioactive sur les écosystèmes locaux.

Des pertes d'animaux de ferme ont été enregistrées dans le village le plus proche de l'accident, alors que pour les villages les plus éloignés aucune mortalité n'aurait été notée avant évacuation. Les doses reçues par la faune et la flore ont été fonction de facteurs agissant en sens divers :

- la composition des rejets : dans le cas de Kyshtym, les émetteurs $\beta^{-}$de courte demi-vie ont été interceptés par la végétation et furent la cause d'une période aiguë d'irradiation ;

- la période de contamination : à l'état de dormance, les dégâts causés sont moindres mais les processus de régénération sont aussi plus lents.

Les premiers effets sur la végétation ont été observés sur les pins (Pinus sylvestris) au printemps 1958 (jaunissement des aiguilles et dessèchement des bourgeons latéraux et terminaux). La mort des pins a été observée en automne 1959 pour une contamination de $180 \mathrm{Ci} \mathrm{km}^{-2}$ $\left(6,6 \mathrm{TBq} \mathrm{km}{ }^{-2}\right)$ de ${ }^{90} \mathrm{Sr}$ et $20 \mathrm{~km}^{2}$ de forêt de pins furent détruits. Une 
croissance anormale de ces arbres fut observée en 1959 (jaunissement, chute d'aiguilles, anomalies morphologiques et physiologiques...) pour une contamination de $40 \mathrm{Ci} \mathrm{km}^{-2}\left(1,5 \mathrm{TBq} \mathrm{km}{ }^{-2}\right)$. Les bouleaux montrèrent une résistance plus grande, ils furent détruits en zone fortement contaminée (4000 $\mathrm{Ci} \mathrm{km}^{-2} ; 148 \mathrm{TBq} \mathrm{km}{ }^{-2}$ ). Des anomalies de croissance se révélèrent également pour des doses plus faibles. Ces différences de radiosensibilité trouvent partiellement leur origine dans la plus grande surface offerte par les aiguilles au dépôt des polluants et aussi dans la sensibilité plus grande des conifères aux rayonnements ionisants. Certaines graminées furent détruites pour des niveaux de contamination de $1500 \mathrm{Ci} \mathrm{km}^{-2}$ $\left(55,5 \mathrm{TBq} \mathrm{km}{ }^{-2}\right)$ et furent remplacées par d'autres ayant un port différent (bourgeons foliaires protégés par le sol). Ces effets se sont manifestés durant 3 à 4 ans et ensuite un processus lentement réversible a été observé. Pour des contaminations inférieures $\left(1000 \mathrm{Ci} \mathrm{km}^{-2} ; 37 \mathrm{TBq}\right.$ $\mathrm{km}^{-2}$ ), on a observé une réduction de la capacité germinative des graines ainsi que des modifications morphologiques tels que gigantisme, chlorose, enroulement des feuilles, réduction du nombre de graines...

Suite à cette pression de sélection, les forêts mixtes pins-bouleaux les plus irradiées ont été transformées en forêts de bouleaux. La destruction totale ou partielle de l'écosystème forestier a entraîné une modification du microclimat sous-jacent (luminosité et pluviolessivage plus importants). Ces modifications ont permis une multiplication de la végétation herbeuse et une modification de la composition floristique. A l'exception de la forêt entièrement détruite, il semble qu'à l'heure actuelle, la nature ait repris ses droits et que la composition floristique ne diffère plus significativement des parcelles non irradiées.

La faune a également souffert de la contamination. Les espèces vivant dans la couche superficielle du sol (litière forestière par ex.) ont été détruites (vers de terre, myriapodes, mites) pour des niveaux de contamination supérieurs à $100 \mathrm{Ci} \mathrm{km}^{-2}\left(3,7 \mathrm{TBq} \mathrm{km} \mathrm{km}^{-2}\right)$. Les espèces plus mobiles et les invertébrés protégés par un abri naturel ont beaucoup moins souffert de la contamination. Les oiseaux et les mammifères n'ont été exposés à des doses létales durant l'automne et l'hiver 1957/58 que s'ils ont hiverné dans des zones à niveau de contamination supérieur à $1000 \mathrm{Ci} \mathrm{km}^{-2}$ (37 TBq km-2). Les oiseaux migrateurs ont donc peu souffert des retombées radioactives car, à leur retour, les niveaux avaient déjà décru d'un facteur 10.

Chez les mammifères vivant dans la zone contaminée, les effets les plus marqués ont été observés chez les rongeurs (souris...). Les effets primaires ont été suivis de modifications de structure des populations et d'une réduction des mécanismes de défense. Cinq ans après l'accident, les populations ne différaient plus des populations témoin. En écosystème aquatique les poissons herbivores (carpe) qui passent l'hiver au niveau des sédiments ont souffert le plus de la contamination. A tous ces effets, il faut signaler que l'abandon des zones contaminées par l'homme, la modification des pratiques agricoles, l'absence de contrôle des populations 
par la chasse par ex. (multiplication des sangliers...) ont également participé à la modification structurale des écosystèmes. Les animaux migrateurs ayant eu la possibilité de passer l'été dans la zone contaminée et d'en sortir avant l'hiver ont posé un problème de radioprotection en cas de chasse en dehors des zones contaminées et contrôlées.

Une première expédition dans la zone contaminée a pu être organisée dans le cadre d'une collaboration entre radioécologistes soviétiques et occidentaux. Des échantillons de sol furent prélevés en mai 1990 dans la zone contaminée par une équipe de scientifiques de la Communauté européenne. L'analyse de ces échantillons a montré que le dépôt de ${ }^{137} \mathrm{Cs}$ a été de deux à trois fois plus élevé que celui calculé sur la base des données connues (accident, retombées dues aux explosions nucléaires atmosphériques et à l'accident de Tchernobyl). II est par conséquent probable que cette région ait été également contaminée par la remise en suspension de particules de sol ou de sédiment du lac Karachay lors d'importantes tornades.

Suite à l'accident de Kyshtym, qui se révèle à l'heure actuelle être du même ordre de grandeur que celui de Tchernobyl, les radioécologistes soviétiques ont acquis une maîtrise du contrôle du milieu et une connaissance élevée des phénomènes régissant le transfert des radionucléides dans l'environnement ainsi que des effets des radiations sur la flore et la faune. II n'est donc pas étonnant de voir avec quelle maîtrise ces scientifiques ont réagi dès après l'accident de Tchernobyl.

L'accident de Tchernobyl a eu lieu le 26 avril 1986, c'est-à-dire au début du printemps alors que celui de Kyshtym s'est passé en automne.

Les plus gros débris et les plus grosses particules se sont déposés près du site alors que les particules plus légères furent transportées à plus grande distance. Cette distribution dans le temps a donné naissance à un dépôt extrêmement hétérogène (en peau de léopard) autour de la centrale accidentée ; les nuages contaminés ont suivi des trajectoires diverses en fonction du temps et les caprices de la météorologie sont également responsables d'une contamination non uniforme des territoires soviétiques situés en dehors de la zone d'exclusion de $30 \mathrm{~km}$ (républiques de Biélorussie, de Russie et d'Ukraine) ainsi que de divers pays européens. Quatre radionucléides furent principalement à l'origine de l'irradiation de la population : ${ }^{131} \mathrm{I},{ }^{134-137} \mathrm{Cs},{ }^{90} \mathrm{Sr}$ et le plutonium $\left({ }^{239-240} \mathrm{Pu}\right)$.

Le bilan officiel du nombre de morts est de 31 ( 2 décès immédiats et 29 décès survenus chez les 237 grands irradiés dans les trois mois qui ont suivi l'accident). Aucun rapport officiel ne mentionne le devenir des personnes et, en particulier, des milliers de soldats qui ont participé aux travaux de décontamination. Parmi les victimes se trouvent également les pilotes d'hélicoptères chargés de couvrir le cratère à l'aide de matériaux divers. Le plus grand danger à court terme pour les populations a été causé par ${ }^{131}$ / transféré rapidement dans le lait par le truchement de 
I'herbe contaminée. La décision d'évacuer la ville de Pripyat a été prise $36 \mathrm{~h}$ après l'accident ; le 28 avril, il fut décidé de créer une zone d'exclusion de $10 \mathrm{~km}$ de rayon et le 2 mai cette distance a été portée à $30 \mathrm{~km}$. L'évacuation de la zone d'exclusion s'est terminée le 6 mai. Au total, 116000 personnes ont été évacuées. D'autres personnes vivant en dehors de la zone d'exclusion ont été évacuées plus tardivement et de nouvelles évacuations sont à l'étude pour que la population ne subisse pas une exposition supérieure à la norme internationale : 0,35 Sv (35 rems) sur une vie entière (70 ans) ; 1000 personnes sont rentrées illégalement dans la zone d'exclusion. Le personnel d'intervention (civil et militaire) était mal entraîné et mal équipé pour se protéger des rayonnements ionisants et les forces d'intervention n'étaient pas préparées à faire face à un accident de cette envergure, les 600000 soldats qui ont participé au nettoyage du site et qui ont été ensuite renvoyés dans leur famille auraient reçu des doses nettement supérieures à $250 \mathrm{mSv}$, dose individuelle acceptée par les autorités dans ce cas.

A l'inverse de Kyshtym, les radionucléides n'ont pas nécessairement été rejetés sous forme directement soluble. Des particules "chaudes" ont été déposées dans la zone proche du réacteur (particules vitrifiées d'un diamètre supérieur à $10 \mu \mathrm{m}\left(10^{-6} \mathrm{~m}\right)$ composées de radionucléides, ou de graphite et de matériaux de construction). La centrale de Tchernobyl se trouve à la frontière de l'Ukraine et de la Biélorussie en zone agricole pauvre (marais de la rivière Pripyat). Les sols y sont surtout sableux et ont une capacité de rétention des radionucléides très faible. L'ensemble de ces facteurs fait que le comportement des radionucléides a été différent dans les deux cas et qu'il est impossible d'extrapoler les résultats obtenus à des zones écologiques différentes. Cette observation met en exergue la nécessité de bien connaître les conditions des milieux potentiellement menacés par des retombées radioactives. Dans la zone d'exclusion, deux écosystèmes ont été particulièrement touchés : la forêt ceinturant le réacteur accidenté et la plaine alluviale située en bordure de la rivière Pripyat.

La zone proche touchée par l'accident de Tchernobyl était couverte à raison de 50 à $60 \%$ de forêts. La forêt rouge (forêt détruite par irradiation et par dépôt de particules radioactives) couvrait 1200 ha et était composée en majorité de conifères (Pinus sylvestris). La dose létale pour les pins fut de l'ordre de 100 Gy (25-30 Gy pour le méristème apical) alors qu'elle aurait été de 50 Gy à Kyshtym (25 Gy pour le méristème apical). La résistance des feuillus a été de 10 à 20 fois supérieure à celle des conifères. Afin de prévenir toute remise en suspension de la radioactivité via les feux de forêts, les arbres furent abattus et enterrés dans d'énormes tranchées servant de sites de stockage provisoires (jusqu'à 800). La revégétation du sol mis à nu a posé d'énormes problèmes aux spécialistes (climat sec et chaud à cette époque). Le recouvrement du sol par un film de plastique enrichi en graines de façon à éviter une évaporation trop forte de l'humidité du sol a permis de restaurer une couverture végétale. Les arbres issus de graines récoltées dans la "forêt 
rouge" présentent tous des anomalies de types divers (chlorose, "balais de sorcière", aiguilles longues et pendantes...). La végétation a aussi été partiellement détruite au-delà de la "forêt rouge" et des croissances anormales, des pins particulièrement, ont été observées à une distance de $20 \mathrm{~km}$ du réacteur accidenté. La demi-vie radioécologique* de la radioactivité interceptée par les arbres a été de 1 mois à Tchernobyl alors qu'à Kyshtym cette demi-période a été plus longue (6-8 mois). Les conditions climatiques sont certainement responsables des différences observées entre les deux sites ("effet saison"). Quatre zones agricoles de forte contamination en ${ }^{137} \mathrm{Cs}$ s'étendent autour de la centrale : supérieure à $40 \mathrm{Ci} \mathrm{km}^{-2}$; de 15 à $40 \mathrm{Ci} \mathrm{km}^{-2}$; de 5 à $15 \mathrm{Ci} \mathrm{km}^{-2}$ et de 1 à $5 \mathrm{Ci} \mathrm{km}^{-2}$ (> 1,5 TBq km-2 ;0,6 à 1,5 TBq km-2 ;0,2 à $0,6 \mathrm{TBq} \mathrm{km} \mathrm{Tm}^{-2} ; 37$ à $185 \mathrm{GBq} \mathrm{km}{ }^{-2}$ ), ce qui correspond à un total de $3010^{6}$ ha. En "forêt rouge", on a mesuré des contaminations allant jusqu'à $400 \mathrm{Ci}$ $(14,8 \mathrm{TBq}) /{ }^{137} \mathrm{Cs} \mathrm{km}^{-2 \star \star}$. Plus aucune culture n'est possible dans les zones contaminées à plus de $40 \mathrm{Ci} \mathrm{km}^{-2}\left(1,5 \mathrm{TBq} \mathrm{km} \mathrm{Tm}^{-2}\right)$; on envisage d'en faire des parcs nationaux fermés au public. Dans les zones de contamination comprises entre 15 et $40 \mathrm{Ci} \mathrm{km}^{-2}\left(0,6\right.$ et $\left.1,5 \mathrm{TBq} \mathrm{km}^{-2}\right)$, on s'oriente vers des formes spéciales d'agriculture (production d'huile, plantation d'arbres pour la fabrication du papier...). Pour les zones comprises entre 5 et $15 \mathrm{Ci} \mathrm{km}-2(0,2$ et $0,6 \mathrm{TBq})$, on pense surtout à l'élevage pour la production de viande.

L'écosystème aquatique proche de la centrale composé des rivières Pripyat, Dniepr et du réservoir situé en amont de Kiev fut également contaminé par le dépôt de radioactivité. Celui-ci est actuellement surtout menacé par une pollution en ${ }^{90} \mathrm{Sr}$ provenant de la zone d'exclusion : ce radioélément est moins fixé par les sols que ${ }^{137} \mathrm{Cs}$ et il est transporté par les eaux à la suite aux inondations du Dniepr ou des hivers rigoureux qui exigent la rupture par explosif de la glace recouvrant la Pripyat (onde de choc provoquant l'inondation des berges).

Divers travaux furent effectués afin d'empêcher une contamination du réservoir par infiltration (sol très léger) et par écoulement lors des fortes pluies. De nombreuses digues (136) furent construites et un mur de $8 \mathrm{~km}$ de long atteignant la couche d'argile profonde $(30-35 \mathrm{~m})$ fut dressé afin d'empêcher toute contamination des eaux s'écoulant vers le Dniepr. Beaucoup de ces digues furent ensuite démantelées car elles ont occasionné l'inondation des forêts contaminées. II n'en subsiste plus que 6 à I'heure actuelle.

Les animaux de ferme n'ayant pas montré de signes cliniques furent évacués et nourris à l'aide de fourrage non contaminé. L'accident s'étant produit au printemps, cette pratique a été rendue difficile par manque de fourrage. Quelques centaines de vaches laitières ont été maintenues à titre expérimental dans la zone des $10 \mathrm{~km}$; elles présentent, à l'heure actuelle, des signes de santé déficiente (manque d'appétit, stérilité, avor-

\footnotetext{
* Temps nécessaire pour que disparaisse la moitié de la radioactivité déposée sur la canopée.
}

** Des teneurs plus élevées ont été relevées dans d'autres zones plus éloignées du réacteur. 
tement). Le gibier en pleine prolifération utilise la zone des $30 \mathrm{~km}$ comme réserve naturelle. La population de sangliers y est passée de 300 en 1986 à plus de 2000 en 1991. Trois meutes de loups occupent actuellement le territoire évacué. Les cervidés capturés dans la zone des $30 \mathrm{~km}$ ne semblent pas présenter d'anomalies ! Par contre, les souris qui résident de manière prolongée dans les $10 \mathrm{~cm}$ de sol superficiel présentent des anomalies chromosomiques des lymphocytes. Certaines modifications des populations d'insectes observées dans la zone pourraient toutefois être dues à l'absence de pratiques agricoles.

Un certain nombre d'échantillons ont été prélevés dans la zone des $30 \mathrm{~km}$ lors des différentes missions effectuées par les experts occidentaux dans la zone d'exclusion. L'analyse de leur teneur en radioéléments par divers instituts et laboratoires a montré combien il est important de mettre au point un programme d'intercomparaison des résultats. Cet exercice est indispensable à la bonne interprétation et à l'étude comparative des résultats publiés par les laboratoires qui s'intéressent au problème de la radiocontamination du milieu. L'analyse des échantillons récoltés en forêt proche du réacteur a aussi révélé que l'activité déposée et interceptée par les arbres se trouve actuellement fixée dans les couches superficielles du sol ( $\left.400 \mathrm{Ci} \mathrm{km}^{-2} ; 14,8 \mathrm{TBq} \mathrm{km}{ }^{-2}\right)$.

Une analyse plus approfondie des propriétés physico-chimiques des différents horizons des sols forestiers est indispensable à l'évaluation du devenir à long terme de la radioactivité ainsi qu'à celle de l'importance du transfert du sol vers les plantes du sous-bois entrant dans la ration alimentaire du gibier et, par conséquent, de celle de l'homme.

Ces deux accidents nucléaires apparaissent comme des catastrophes radioécologiques très graves. La seconde (Tchernobyl) s'inscrit, de plus, dans un contexte politique complexe et ce dans le cadre d'un pays connaissant des difficultés économiques importantes. Ces conditions conjuguées à une situation sanitaire désastreuse rendent très difficile l'estimation de l'impact de l'accident sur les populations. A cela on peut ajouter que l'état initial du milieu (pollution par les rejets industriels et par l'emploi de pesticides et d'engrais en agriculture) complique fortement l'étude de l'impact de Tchernobyl sur l'état de santé des communautés touchées par la radiocontamination.

Les modifications structurales des écosystèmes locaux reflètent, toutefois, l'ampleur de ces catastrophes et le territoire de Tchernobyl constitue le meilleur champ d'expérience permettant de mettre au point les modèles de transfert à long terme de la radioactivité, étudier la radiosensibilité des écosystèmes et, ainsi, de parfaire les plans d'urgence en cas de contamination nucléaire du milieu. Aux problèmes écologiques mentionnés ci-dessus, il faut ajouter tous les travaux visant à réhabiliter les zones sinistrées et ainsi à permettre une diminution du transfert de la radioactivité dans les divers écosystèmes touchés par la radiocontamination. L'étude de contre-mesures en cas d'accident nucléaire constitue également un important volet de la recherche radioécologique. 